\title{
ARMA SUMPSERIT(LUCAN. 7, 259-260). MARADONA, MALVINAS Y EL ARTE DE LA CAUTA CRÍTICA
}

\author{
Arma sumpserit (Lucan. 7, 259-260). Maradona, Malvinas and the art of safe \\ criticism
}

\author{
Gabriela Andrea Marrón* \\ UNS-CONICET \\ marron.gabriela@gmail.com
}

Palabras clave

Homero;

Lucano;

Maradona;

estrategias discursivas;

ambigüedad

\section{Resumen}

El propósito de este trabajo consiste en estudiar las estrategias discursivas utilizadas por Frederick Ahl en el artículo titulado "A mão de Deus: Diego Maradona e a natureza divina da trapaça na Antiguidade Clássica", estableciendo sus convergencias con ciertos recursos poéticos y procedimientos retóricos descriptos por él en sus investigaciones sobre la literatura clásica. Intentaré demostrar que, para estudiar la heroificación del gol de Maradona contra Inglaterra, el autor urde un contexto interpretativo mayor, donde las referencias mitológicas funcionan como veladas alusiones al conflicto de Atlántico Sur.

\begin{abstract}
The purpose of this research article is to study the discurse strategies used by Frederick Ahl in the academic paper entitled "The hand of God: Diego Maradona and the divine nature of cheating in Classical Antiquity", establishing their convergences with certain poetic resources and rhetorical devices described by him in his researches on classical literature. I will try to prove how, in order to study the heroized goal of Maradona against England, the author sets up a larger interpretative framework, in which mythological references function as veiled allusions to the South Atlantic conflict.
\end{abstract}

Keywords

Homerus;

Lucanus;

Maradona;

discurse strategies;

ambiguity 


\title{
Arma sumpserit (Lucan. 7, 259-260). Maradona, Malvinas y \\ el arte de la cauta crítica ${ }^{1}$
}

\author{
Derrota, tú has creado \\ la fábula del mar \\ $y$ de sus monstruos.
}

Aldo Oliva

He procurado titular este artículo de una manera lo suficientemente extraña como para despertar la curiosidad de potenciales lectores, porque fue precisamente la mención de Maradona en el título de otro trabajo de investigación lo que me llevó, no solo a comprender la relación existente entre la naturaleza divina de la trampa en la Antigüedad clásica y el primer gol de Argentina contra Inglaterra en la Copa Mundial de Fútbol de 1986, sino también a elaborar la hipótesis de lectura que vertebra este escrito. Me refiero al trabajo titulado A mão de Deus: Diego Maradona e a natureza divina da trapaça na Antiguidade Clássica, que el profesor Frederick Ahl envió en agosto de 2014 a la revista Archai, de la Universidad de Brasilia, y fue luego publicado en el número correspondiente al primer semestre de 2015. Leer al autor de Metaformations es siempre una grata experiencia de aprendizaje, a la que en este caso se sumó la singularidad del tema y del lugar de enunciación: el reconocido clasicista, formado en los claustros de Cambridge y de Texas, no solo proponía estudiar las representaciones de la trampa en el arte y la literatura grecorromana, para demostrar que aquel gol de Maradona con la mano resultaba comparativamente trivial; sino que además elegía hacerlo en Brasil, meses después de que finalizara el primer Campeonato Mundial de Fútbol desarrollado en Sudamérica desde 1978. Y como si esa articulación de coordenadas no bastara para despertar la curiosidad, en la nota al pie inicial, el autor mencionaba haber presentado una versión preliminar del trabajo en julio de 2012, durante la conferencia anual de la 'Sociedad Literaria de Londres', cuyo eje temático, relacionado con los Juegos Olímpicos que tendrían lugar al mes siguiente en suelo inglés, fue 'Deportes, Juegos y Pasatiempos' (Ahl, 2015a, p. 11, n. 1).

\section{Curiouser and curiouser}

En medio del enrarecido clima diplomático reinante entre Argentina e Inglaterra a causa del trigésimo aniversario de la Guerra de Malvinas, Frederick Ahl había disertado acerca de aquel tramposo gol en la capital británica; tres años después, una versión escrita de esa conferencia sobre la dimensión heroica de Maradona aparecía publicada en el país de Pelé. Más allá de la curiosa insistencia en elegir horizontes geográficos y culturales potencialmente adversos para referirse al tema, lo que me sorprendió, al realizar la primera lectura global del artículo, fue la idea de que muchos argentinos pudieran considerar que la mano de Dios, en el campo de juego de 1986, fuera equivalente a la mano que el Reino Unido recibió de Ronald Reagan en el campo de batalla de 1982. La comparación

1. Este trabajo ha sido escrito en el Centro de Estudios de Filología Clásica Antigua y Medieval del Departamento de Humanidades de la Universidad Nacional del Sur, en el marco de proyectos de investigación financiados por la ANPCyT (PICT 2016 No 1012), la SGCyT de la UNS (PGI 24/I 271) y la SGCyT de la UNNE (PI 17H014). 
no solo resultaba curiosa porque en nuestro imaginario colectivo el anverso de aquella trampa haya sido siempre el hundimiento del Crucero ARA General Belgrano (cfr. Steinsleger, 02/12/2020; Mateo, 02/02/2021), sino porque además el autor luego desestimaba dicha relación de equivalencia supuestamente establecida por el punto de vista argentino: "Wrongly so; it has become increasingly clear that Reagan did not approve of Thatcher's surprisingly forceful military response to the Argentinian invasion; see (Aldous, 2012), especially p. 71-96" (Ahl, 2015a, p. 13, n. 7). Sin embargo, al intentar consultar esa referencia, observé algo incluso más curioso. La numeración de las páginas del libro de Aldous (2012a), titulado Reagan and Thatcher: The Difficult Relationship, parecía desplazada, ya que no correspondía al inicio y al final de un capítulo puntual, ${ }^{2}$ sino a un segmento que abarcaba desde la tercera página del capítulo cuarto hasta la segunda del quinto. La primera línea de la página 71 comenzaba con las palabras "destroy the weak", y en el centro de la página 96 podía leerse:

[At the White House], reaction to the sinking of the Belgrano and the Sheffield was one of astonishment and horror. 'The stance of this two disputants increasingly resembles that of a couple of staggering streetfighters, spastically-swinging at each other while blinded into fury by the flow of their own blood', national security staff James Rentschler recorded in his diary. An NSC paper, The Falkland islands: What Now? What Next? was not as colorful, but its incomprehension at what was happening was not less clear. 'The sinking of the Belgrano and the Sheffeld bring the South Atlantic conflict to an alarmingly new and perhaps desperate stage, one which throws into sharper relief the negative strategic factors which the US will increasingly confront as the hostilities persist', the paper bluntly stated. 'We are in a situation where only an act of sanity may now save not only the belligerents themselves from further loss, but larger US interests as well.'

Como es posible inferir, la aparente errata numérica era lo suficientemente trivial como para ser desestimada y, a la vez, lo bastante precisa como para poner en duda el azar. Volví a verificar la edición citada en la bibliografía, y fue recién entonces cuando noté la discrepancia. Allí se indicaba que el libro de Aldous había sido editado por Random House en Nueva York (Ahl, 2015a, p. 18), pero el volumen que yo tenía a la vista había sido impreso en Londres por Hutchinson, Random House (Aldous, 2012a). El azar parecía imponerse. Sin embargo, cuando algunos días después pude interrogar la versión neoyorquina, donde las páginas 71 y 96 corresponden al comienzo y al final del capítulo cuarto, observé que la edición no era de Random House, sino de W. W. Norton E Company (Aldous, 2012b). Me sentí una suerte de versión vernácula de Bioy Casares ante el volumen XXVI de la falaz AngloAmerican Cyclopaedia que reproducía la décima edición de la Encyclopaedia Britannica.

\section{Sinking the unthinkable}

Como sostiene Frederick Ahl (2015b, p. 240), lo 'impensable' es una categoría disciplinar predominante y muy rara vez discutida, que no constituye un conjunto de ideas imposibles de pensar (si las hay), sino de formas de pensar que el mundo académico insiste en desestimar. Ante una referencia

2. La página 71 forma parte del cuarto capítulo, titulado "Little Ice-Cold Bunch of Land", que se extiende de la página 69 a la 94; la página 96, en cambio, forma parte del siguiente, cuyo título es "Even More a Wimp than Jimmy Carter" y comienza en la página 95. 
bibliográfica problemática, como la descripta en el apartado precedente, deberíamos pensar que se trata de una ecdótica confusión de ciudades y editoriales. Lo impensable, en cambio, sería aproximarse a esa errata como si fuera uno de los recursos que posee el texto para llamar la atención sobre sí mismo; al fin y al cabo, si en los escritos académicos no predomina la función poética, es precisamente porque "la primacía de la función poética sobre la función referencial no elimina la referencia, pero la hace ambigua" (Jakobson, 1975, p. 382-383).

Curiosamente, en el universo de las referencias bibliográficas del artículo que nos ocupa impera una cautelosa ambigüedad. Por ejemplo, tras afirmar: "The ancient tale of Olympia is as much the tale of powerful dynasties, wars, politics, cheating, and treachery, as it is of athletics" (Ahl, 2015a, p. 15), el autor inserta una nota al pie en la que agrega: "So too in the modern Olympics, most notably those of 1936. See (Mandell, 1987); (McSmith, 2008)”. En este caso, la ambigüedad supone una confusión de independencias y no de páginas, ya que si bien la nota de opinión de Andy McSmith fue publicada por primera vez el 8 de abril de 2008, como consta en el apartado de referencias bibliográficas (Ahl, 2015a, p. 19), no apareció en el periódico británico The Independent, sino en Irish Independent, un diario irlandés fundado en 1905 (cfr. McSmith, 08/04/2008). Podría tratarse de otra simple confusión, porque de hecho esa misma nota de opinión volvió a ser publicada, meses antes de los juegos Olímpicos de Londres, precisamente en The Independent (cfr. McSmith, 23/10/2011). Sin embargo, de los múltiples goles cometidos con la mano a lo largo de la historia del fútbol, Frederick Ahl (2015a, p. 13) elige detenerse precisamente en la del francés Thierry Henry: "Maradona was not the first, and far from the only, footballer to score a goal with his hand. A similar, though less direct, incident eliminated Ireland from the final rounds of the 2010 World cup". Y para refrendar las palabras del futbolista citadas a continuación, hay una referencia bibliográfica que, a diferencia de la anterior, sí incluye el enlace al artículo periodístico correspondiente (Ahl, 2015a, p. 13, n. 9; y p. 19). Si la confusión de independencias en torno a la duplicada nota de $\mathrm{McSmith}$ no es producto del azar, ¿podía relacionarse con la posición adoptada por la República de Irlanda tras el hundimiento del Crucero ARA General Belgrano? (cfr. Galego-Díaz, 04/05/1982; O’Connell, 29/12/2012; 02/04/2019). ${ }^{3}$

Lo que Frederick Ahl hace en su artículo sobre Maradona y la mano de Dios es enlazar ese episodio futbolístico en una trama interpretativa mayor, donde las narraciones míticas, relacionadas con el origen de las competencias olímpicas y con los juegos fúnebres presentes en los textos épicos, cumplen un papel fundamental. Y, puesto que él mismo afirma que la presencia de alusiones a las circunstancias contemporáneas de los escritores en sus textos constituye uno de los 'impensables' más amplios que debemos sortear al estudiar la literatura basada en mitos griegos (Ahl, 2015b, p. 241), trabajaré a partir de la hipótesis de que lo mismo sucede con su tratamiento del mito en The hand of God: Diego Maradona and the divine nature of cheating in Classical Antiquity. Para ponerla a prueba, en los siguientes apartados analizaré algunos pasajes de ese artículo, donde es posible observar la reproducción de estrategias poéticas y retóricas descriptas por el autor a lo largo de su vasta producción académica.

3. Guillermo Brown, el Primer Almirante de la fuerza naval argentina, había nacido en el Reino de Irlanda (cfr. Moreno, 2010, p. 115-122); en 1983, la banda de rock irlandesa The Wolfe Tones, enlazó simbólicamente su nombre con las Islas Malvinas, en la canción titulada Admiral William Brown (cfr. Baños Pozzati, 06/10/2020). 


\title{
Don't think of an elephant
}

Como los juegos de palabras suelen resultar incompatibles con las representaciones de 'lo clásico' predominantes en nuestro campo de estudio, quienes intentan demostrar su presencia en las obras literarias grecolatinas siempre batallan cuesta arriba (cfr. Ahl, 1985, p. 18). La comunidad científica experimenta cierto rechazo ante la idea de que un escritor serio pueda balancear su discurso en torno al doble sentido de un término y jugar ambiguamente con las posibilidades semánticas que le ofrece el lenguaje (cfr. Fantham, 1995, p. 372; y Ahl, 2011, p. 36). Por lo tanto, sería 'impensable' que algo así ocurriera en un artículo de investigación sin generar una suerte de ruptura en el pacto discursivo del género (cfr. Ludmer, 2000, p. 68, n. 11); excepto, acaso, cuando quien nos invita a pensar de esa forma es el mismo autor del texto: "A pun is a phoneme or series of phonemes which has no simple meaning. Before concluding that a writer intends only one of its meanings, we should carefully examine the possibility that he intends more" (Ahl, 1988, p. 24). Observemos, por ejemplo, lo que sucede en el siguiente fragmento de su artículo sobre Maradona y la mano de Dios (Ahl, 2015a, p. 12-13):

\begin{abstract}
Winning is ultimately what matters in games and warfare, ancient and modern; and, in practical terms, winning has little to do with morality. While rules and conventions have been devised to make competition less brutal, victory and defeat establish innocence and guilt respectively, as Lucan's Julius Caesar cynically points out to his troops that the battle of Pharsalus will establish "who took up arms more justly; this battle will make the loser guilty: quis iustius arma,/sumpserit; haec acies victum factura nocentem est.' (Pharsalia 7.259-60). Success was routinely construed as evidence of divine favor by both ancient Greeks and ancient Romans. Victrix causa deis placuit sed victa Catoni, Lucan observes editorially of the Caesarian civil war (Pharsalia 1.128): winning won the gods' approval, losing won (the good man) Cato's approval. Civil war in his eyes was a criminal activity and it was better to lose such a war than to win it. But Lucan's notion of 'moral victory' has acquired over the centuries a curiously GENERAL currency. Even nowadays, when a person is said to have won the moral victory, in battle or on the playing FIELD, he or SHE has GENERALLY lost whatever the contest is. It is the winner the gods approve. How he wins is less important.
\end{abstract}

El léxico utilizado al comienzo del párrafo contribuye a conjurar la presencia de las dos embarcaciones perfiladas al final. El buque británico Sheffield emerge a partir del 'campo' (field) de batalla y del uso inclusivo de los pronombres (he or she). ${ }^{4}$ Nótese que las letras de los dos monosílabos centrales (he or) forman, además, una suerte de anagrama votivo: hero ('héroe'). Y como en inglés el género gramatical de los barcos no era neutro, sino femenino (cfr. Deutscher, 2010, p. 226-227), la presencia del pronombre she en ese contexto resulta reveladora. El Crucero ARA General Belgrano, a su vez, surge no solo por el eco de general-ly y general, sino también por la anteposición del adverbio curiously ('curiosamente'), cuya pronunciación apresurada evoca el sustantivo cruiser ('crucero'). La palabra winning ('ganar)' se repite tres veces; y a la presencia inicial del sustantivo morality ('moral') se suma la duplicación del sintagma moral victory ('victoria moral'), normalmente ligado a la derrota en combate, como en el siguiente fragmento de una nota publicada en el periódico londinense The Economist, el 8 de mayo de 1982 (cit. en Moro 1989, p. 190; 1985, p. 288; y 2005, p. 170): "Great

4. Cfr. Ahl (2015b, p.14): "Shrewd fouls and sheer luck larger LOOM larger in soccer than in most other sports". El

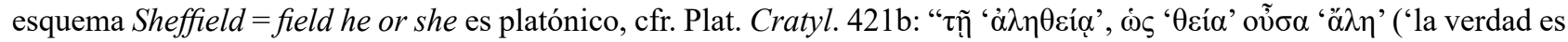
un viaje divino'); y Ahl (2002, p. 129-130). 
Britain was elated with her early successes; then came Argentina's first great moral victory (the sinking of the Belgrano), followed by her first naval victory (the sinking of the Sheffield)".

Incluso más interesante es el juego etimológico que insinúa el autor al agregar una coma al final del primero de los dos versos de Lucano citados (cfr. Shackleton Bailey, 2019, p. 172), cuyo indicio es: 'Lucano observa, desde el punto de vista editorial...' (Lucan observes editorially...). Antes de la batalla de Farsalia, al dirigirse a sus soldados, César sostiene que ese día probará quién 'habrá tomado' (sumpserit) las 'armas' (arma) 'con mayor justicia' (iustius), y que 'esa batalla' ( haec acies) 'ha de convertir' (factura) al 'vencido' (victum) en 'culpable' (nocentem). Sin embargo, quien parece operar allí como un editor es el autor, al introducir la coma sobrante y guiar nuestra atención hacia el verbo, sumpserit, que resulta etimológicamente pertinente en castellano:

SUMIR, del lat. sūměre 'tomar', que a veces se aplicaba a los alimentos, y de aquí pudo pasar al sentido de 'tragar' y luego 'hundir bajo tierra o bajo el agua'; o quizá de su derivado absūměre, que ya significaba propiamente 'tragar', 'devorar'y 'aniquilar'. (Corominas, 1983, p. 335, 1. 26-32)

Por otra parte, también es significativo que, en un artículo donde se exploran las resonancias simbólicas de un gol convertido con la mano, el autor elija citar sólo los dos primeros versos de un pasaje cuyo sentido se completa con los tres siguientes, sumamente esclarecedores en cuanto al parámetro de justicia reinante en la distribución de las responsabilidades:

si pro me patriam ferro flammisque petistis, nunc pugnate truces gladioque exolvite culpam: nulla manus, belli mutato iudice, pura est.

(Lucan. 7, 261-263)

Si por mí la patria a hierro y fuego atacaron, ahora combatan despiadados y con la espada deslíguense de la culpa: ninguna mano está limpia si cambia el juez de la contienda. ${ }^{6}$

En latín, manus significa 'mano', pero también 'tropa' o 'grupo de soldados' (cfr. ThLL v. 8, p. 342, c. 366, 11. 47-50). En inglés, dentro del léxico náutico, algo similar sucede con el vocablo hand, que además de 'mano' significa 'miembro de la tripulación de un barco' (OED ${ }^{3}$, s.v. hand, a. 18); de allí el juego de palabras propuesto por Roger Waters en Get your filthy hands off my desert ('Saca tus sucias manos de mi desierto'), cuatro años antes de que Maradona convirtiera sus dos goles contra Inglaterra:

Galtieri took the Union Jack.

And Maggie, over lunch one day,

took a cruiser with all hands.

5. Sobre los juegos verbales con la etimología de varias lenguas distintas, cfr. Ahl (1985, p. 60-63) y, particularmente, Ahl (2007, p. 415): "The Italy Virgil recalls is not limited to its triumphant Latin speakers, even if the names must be carefully encrypted".

6. Cfr. Ahl (1985, p. 99): "Only one word, Servius observes, is changed: uno tantum sermone mutato. But that change alone is needed to recall the story and to redirect it, as in Horace's famous satirical mot: "change the name and the story is about you" (mutato nomine, de te / fabula narratur [Satires 1.169-70])". Todas las traducciones incluidas en este trabajo son de mi autoría. 
Apparently, to make him give it back

Galtieri tomó la bandera del Reino Unido. Y Maggie, durante el almuerzo un día, tomó un crucero con toda la tripulación a bordo, supuestamente, para obligarlo a devolverla. ${ }^{7}$

Una de las principales contribuciones de Frederick Ahl al estudio de la literatura clásica ha sido, precisamente, demostrar que los juegos de palabras no son simples ornamentos poéticos, accidentes lingüísticos o despliegues de erudición alejandrina, sino también polisémicas estrategias formales, muchas veces orientadas a desestabilizar las certezas políticas imperantes (cfr. Ziogas, 2016, p. 2-3). No obstante, como las distintas representaciones del deporte y la guerra se han imbricado mutuamente a lo largo de la historia, también es normal que determinadas palabras conserven, como en este caso, inesperadas e involuntarias correspondencias simbólicas con otras.

\section{On the pallid bust of Pallas just above}

Al comienzo del artículo, el autor niega que las trampas y las discusiones en torno a lo sucedido en alguna competencia deportiva, ya sea dentro o fuera del campo ficcional, constituyan una novedad. Para ejemplificarlo, señala que Clitias, el pintor que ilustró con distintas escenas mitológicas la cratera conocida como 'Vaso François', se ocupó de consignar cuidadosamente el nombre de los cinco participantes en la carrera de carros de los juegos fúnebres en honor de Patroclo (cfr. Wachter, 1991, p. 89). Solo uno de los cinco guerreros representados allí, Diomedes, forma parte de los competidores presentes en la versión homérica del episodio: Odiseo y Automedón no intervienen en esa prueba, y a los dos soldados restantes, Damasipo e Hipotón, no se los menciona nunca en la Ilíada. No sabemos por qué Clitias juzgó necesario consignar los nombres de cada participante, "[he] may be suggesting things weren't the way the new editions of Homer suggests", sugiere a su vez el autor (Ahl, 2015b, p. 11); y nos recuerda que, en la Ilíada, la carrera de carros genera discusiones tanto entre dos espectadores, como entre dos contendientes. Finalmente, luego de contar que Menelao había llegado tercero y acusado a Antíloco de haberle cerrado arteramente el paso en un tramo del recorrido, asevera: "The earliest account of a chariot race in Western literature, then, is marked by cheating” (Ahl, 2015b, p. 12). La cadena de afirmaciones y rectificaciones que comienza entonces en el trabajo acerca de la maniobra de Antíloco tiene muy poco que envidiarle a las repercusiones periodísticas de la mano de Dios.

Primero, el autor sostiene que los reclamos de Menelao se hallan respaldados por la narración homérica, pero en la nota al pie incluida para refrendarlo (cfr. Ahl, 2015b, p. 12, n. 4) remite a un artículo de investigación que postula una tesis diferente:

From Menelaus' point of view Antilochus cheated, but the more objective view is that he used skill to compensate for his slower horses (v. 515), just as Nestor had advised him. The vagueness of the description of the actual events allows the poet to suggest that both sides may have some justification for their positions and to preserve the dignity and honor of all involved. (Gagarin, 1983, p. 39)

7. Incluida en The final cut, un disco de Pink Floyd de 1983; sobre la relación de Roger Waters con la Guerra de Malvinas, cfr. Guesdon y Margotin (2017); Waters (05/03/2012); y Goñi (06/03/2012). 
Más adelante, lamenta que los clasicistas tiendan a suavizar las antiguas narraciones de 'acciones criminales' (criminal activity) ${ }^{8}$ y de 'trampas' (cheating), tal como hace David Kyle (2007), en su libro sobre el deporte y el espectáculo en la Antigüedad, cuando habla de 'atropellar y jugar al límite de las reglas', o reduce a una 'peligrosa maniobra de conducción' la 'falta'9 cometida por Antíloco (Ahl, 2015b, p. 15):

Donald Kyle, for example, talks of "bustling and gamesmanship" rather than of "cheating" and describes Antilochus' foul on Menelaus as "dangerous driving". Even the great Sir Richard Jebb claimed just two years before the modern revival of the Olympics in Athens in 1896 that Pindar's version of the Pisatan tradition is "the older and nobler form of the myth" (italics mine).

Lo extraño es que, una vez más, las referencias bibliográficas -que en este caso se encuentran en las notas 16: “See (Kyle, 2007, p. 130; 59)”, y 17: “See (Jebb, 1894, [p. 75])”- resultan inexactas. En primer lugar, porque David Kyle dista de idealizar el pasado deportivo de la Antigüedad, ${ }^{10}$ incluso en el apartado que comienza con la expresión tomada por Ahl de la página 130: "Hustling and gamesmanship are not new in sport", cuyo título es "Opportunism and Corruption”. En segundo lugar, porque al final de la página 59 del libro de Kyle, en vez del sintagma "dangerous driving", hallamos otro que, pese a ser similar, no se condice con las comillas de Ahl (Kyle, 2007, p. 59): "Raising issues about fair play and obsession with success, Antilochos nearly causes an accident by driving dangerously”. Y, en tercer lugar, porque el dato más relevante que aporta esa cita -donde el adjetivo deviene adverbio- es la inesperada aparición de Palas, ayudando a Diomedes a ganar la homérica carrera de carros, justo arriba, en el párrafo anterior:

Descending to help the frontrunner Diomedes, Athena retrieves his whip after Apollo made him lose it, and she then breaks the chariot yoke of Diomedes' challenger Eumelos (23.389-92). She goes on to inspire Diomedes and his horses to win (23.400). With the gods playing favorites and fouling charioteers, must the bumans act like perfect sportsmen?

No parece haber gran diferencia entre la imagen de Atenea, hundiendo a Áyax Telamón en los sucios restos de los sacrificios para que Odiseo pueda ganar la carrera a pie (Il. 23, 768-784), y la intervención previa de la misma diosa, destrozando el yugo de los caballos de Eumelo, para que Diomedes triunfe (Il.23, 388-400). Y, sin embargo, páginas atrás, luego de afirmar que el relato más antiguo de una competencia de carros en la literatura occidental había estado marcado por la trampa, el autor aclaraba (Ahl 2015b, p. 12):

But in the footrace at the same Homeric games, there is even some divine cheating: the goddess Athena intervenes, in response to Odysseus' prayer for help, and sends Salaminian Ajax sprawling face down in the fitlhy waste of sacrificial bulls to prevent him winning and thus give victory to

8. Cfr. la presencia del mismo sintagma en el ya citado pasaje sobre la victoria moral; aquí aparece por segunda vez, yuxtapuesto al término trampa.

9. Cfr. Willcock (1988-1989, p. 5): “in Antilochus' action out on the course [we see] the soccer or rugby player who impedes an opponent with a straight run for the goal"; y Ahl (2015, p. 14); allí, dentro de un mismo párrafo, utiliza en tres oportunidades el gerundio fouling y en cuatro el sustantivo foul (tres de ellas en plural, una en singular), siempre para referirse al fútbol.

10. Cfr. Kyle (2007, p. 199): "Ancient athletes cheated earlier and more often than purists would like". 
Odysseus (Iliad 23.768-784). If the gods cheat to help their human favorites, can cheating itself be wholly unacceptable even on a solemn occasion honoring a dead warrior?

Tras nunca explicitar que Diomedes había recibido ayuda de Atenea en la carrera de carros, e insistir en las distintas interpretaciones de la maniobra de Antíloco, el autor afirmaba que, 'sin embargo' (but), en la carrera a pie, había 'incluso' (even) algunas trampas divinas, induciéndonos así a olvidar la otra la mano de Dios. Por lo tanto, la irónica pregunta de Kyle respecto a si hay que jugar como honorables ladies \& gentlemen, aunque los dioses expresen leoninos favoritismos y cambien las reglas de juego, adquiere cierto valor metapoético al ser reformulada por Ahl: 'Si los dioses meten la mano para ayudar a sus mortales preferidos, ¿puede ser totalmente inaceptable hacer trampa incluso en la solemne ocasión de honrar a un caído en combate?. ${ }^{11}$ Recurriendo, entonces, a un cuidadoso entramado de aparentes errores en las notas al pie y en las referencias bibliográficas, ${ }^{12}$ el autor nos permite vislumbrar el otro lado del tapiz. Del mismo modo que ha logrado silenciar la asistencia externa recibida por Diomedes en la carrera de carros, centrando su narración en las respectivas dimensiones humana y divina de las trampas de Odiseo y de Antíloco, también ha aparentado omitir el precedente de mayor resonancia en torno al gol de Maradona con la mano. Y así como la musa del texto homérico logra convencer a su auditorio externo e interno de dos nociones opuestas a la vez (Ahl y Roisman, 1996, p. 164), también Ahl reconfigura las convenciones genéricas del artículo de investigación, adoptando como arma las mismas estrategias formales y discursivas que lo constituyen. Por eso, cuando a continuación de las dos citas textuales de Kyle, consigna cuidadosamente que fue él quien destacó las palabras "older" y "nobler" en la expresión del Caballero Richard Jebb ("italics mine", Ahl, 2015b, p. 15), el efecto que logra crear es doble: por un lado, apela al criterio de autoridad y se apoya en la categoría disciplinar de lo 'impensable'; por otro, interpela nuestro escepticismo y sugiere la posibilidad de ver algo más que imprecisiones o casualidades en las otras referencias bibliográficas. ${ }^{13}$

\section{A fatal and cruel hand}

La polémica entablada por Ahl a partir de la cita de Jebb se relaciona con el valor y la cronología asignada a las distintas versiones míticas en torno al origen de los Juegos Olímpicos. Para comprender mejor a qué se refiere, hay que buscar el emplazamiento original del sintagma “dangerous driving", que, si bien no se encuentra literalmente en la página 59 del libro de Kyle, puede recuperarse a partir de la referencia a un trabajo de Malcom Willcock, incluida al comienzo del tercer capítulo (cfr. Kyle, 2007, p. 54, n. 1 y p. 354). En dicho artículo de Willcock (1973, p. 1), leemos:

Antilochus enters the chariot race with the slowest horses; politely stands and listens to a lengthy speech of advice from his father; waits his time in the race, and then gets past Menelaus at a place

11. Cfr. Ahl (2007), particularmente, p. 169 y 181.

12. Cfr. Ahl y Roisman (1996, p. 163): "[He] is a schemer. We must see what indications of schematic purpose subtend these adjustments".

13. Cfr. Ahl (1984a, p. 197): "[Ancient rhetoricians] suppose in an audience the desire to believe; we suppose a tendency to disbelieve (...) [they] would probably have been amused by our concern for the intellectual skeptic who must be induced to suspend disbelief". 
where two chariots cannot safely run abreast, so that Menelaus holds back. (...) Antilochus gets his second prize. But then up comes Menelaus, full of annoyance, and enters an objection on the charge of dangerous driving.

A diferencia de Michael Gagarin (1983), que analiza cuidadosamente la descripción integral de la escena, y de Frederick Ahl (2015, p. 12) que, tras describir la intervención divina de Atenea para favorecer a Odiseo, señala la curiosa interpretación de Antíloco ante lo sucedido, ${ }^{14}$ David Kyle (2007, p. 59) simplemente afirma que este hizo trampa, perdiendo de vista tanto la perspectiva enunciativa, como la posición de poder de quien realiza esa acusación en la Ilíada. Nótese, por ejemplo, que Menelao

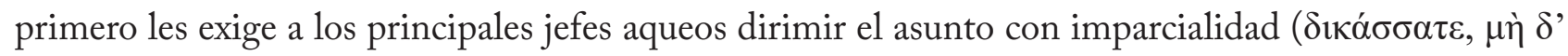
$\dot{\varepsilon} \pi$ ' $\rho \omega \gamma \tilde{n}, I l .23,574$ ), pero finalmente se autoproclama juez (y parte) en la contienda.

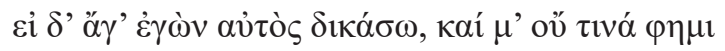

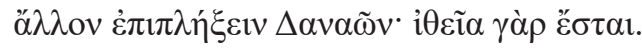

(Il. 23, 579-589)

Ea, vamos, yo mismo dictaré sentencia, y tengo para mí que de los Dánaos ningún otro contragolpeará, porque ha de ser recta.

Mutato iudice, acaso Antíloco no hubiera tenido que disculparse con tanta cortesía. Por otra parte, hablar ante los poderosos requiere ciertos recursos discursivos que ese personaje despliega con habilidad. Finalizada la carrera a pie, Antíloco afirma que los dioses honran a los hombres que tienen

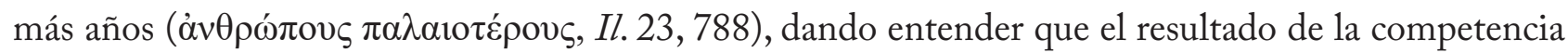
ha reflejado, con justicia, la edad relativa de los competidores. Pero luego no solo aclara que él es el menor de los tres, una información que no se condice con el desenlace de la carrera, donde Antíloco obtiene el segundo puesto a causa del tropiezo de Áyax; sino que además logra elogiar a Odiseo de un modo tan extraño ${ }^{15}$ como para que Aquiles, complacido acaso no solo por la comparación, le sume medio talento al premio que había obtenido:

14. Cfr. Ahl (2015b, p. 12): "And the third place competitor (the same man who cut off Menelaus in the chariot race) observes that Odysseus is of an older generation and that the gods respect seniority". No decir el nombre contribuye a destacarlo, cfr. Ahl (1984a, p. 192-196); Ahl (1984b, p. 83): "Silence is an appropriate term for speech in which what is meant is not said, but left for the listener to discover"; y Ahl (2010, p. 13): "[Quintilian] is, of course, putting us on notice that if we cannot read his silence we will not understand the full implications of the text. He is writing aperte".

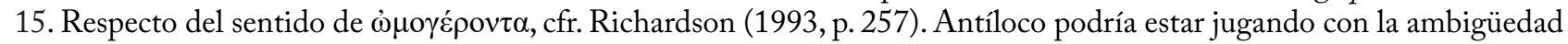

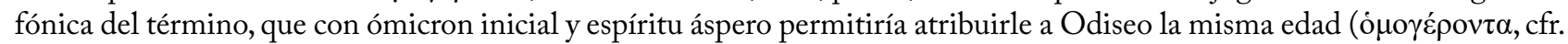

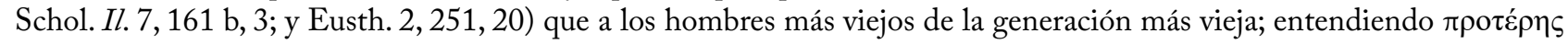
$\gamma \varepsilon v \varepsilon \tilde{\eta} \zeta$ como genitivo epexegético de $\pi \rho \circ \varepsilon \varepsilon \rho \omega v$ à $v \rho \rho \omega ́ \pi \omega v$. La diferencia de espíritu y de cantidad vocálica obstaculizan esa lectura desde el punto de vista escrito, pero para la oralidad del rapsoda (como para la de Antíloco) bastaría con una

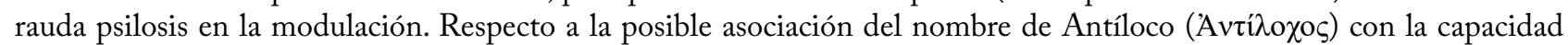

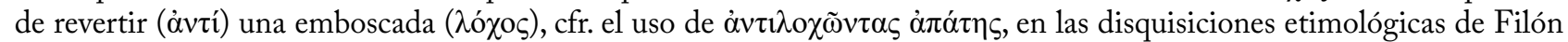
de Alejandría (Phil. somn. 2, 31-40), y lo señalado sobre el nombre de Orsíloco en Ahl (2002, p. 120): "the elements of

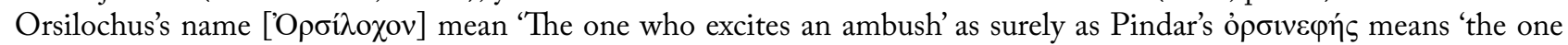

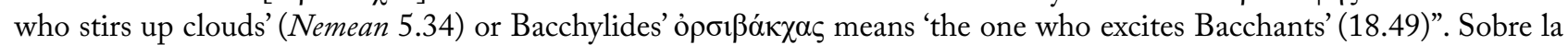
relación de Antíloco con Aquiles, creo que sigue siendo relevante el análisis de Willcock (1973). 


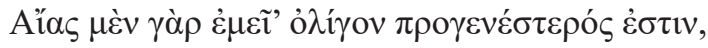

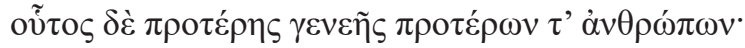

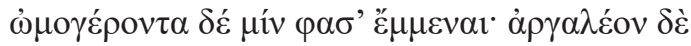

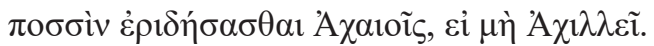

Porque Áyax es apenas mayor que yo, pero este [i.e., Odiseo] es de la generación anterior de los anteriores mortales. Afirman que es un bisoño veterano, pero para todos los aqueos es difícil competirle con los pies, excepto para Aquiles.

Frederick Ahl ha insistido, en distintos trabajos, ${ }^{16}$ acerca de la necesidad de mirar con cuidado el contexto enunciativo en que los personajes narran determinada versión de un mito, justamente porque la creación poética requiere trabajar de manera simultánea a partir del conocimiento de las otras variantes míticas que los destinatarios intra y extradiagéticos podrían llegar a tener. Tanto su análisis de las distintas historias narradas por Odiseo a los diferentes personajes del poema homérico, como su lectura del episodio de Céfalo y Procris en las Metamorfosis de Ovidio, o del relato de Eneas a Dido en la epopeya virgiliana son ejemplos representativos de la forma de acercamiento crítico a la literatura clásica propuesta por este autor. En tal sentido, lo que Frederick Ahl hace, en su artículo sobre Maradona y la mano de Dios, es proceder del mismo modo que Quintiliano en sus Instituciones Oratorias,${ }^{17}$ indicándonos no solo cómo recorrer las asociaciones que ofrece la polifonía de los clásicos, sino también cómo desmontar los discursos que recurren a esos mismos procedimientos con el objetivo contrario. En tanto lectores activos, como interlocutores críticos -y, potencialmente, cómplices- Ahl nos propone observar el funcionamiento de un artefacto académico que subvierte las reglas, pero a la vez apela a nuestro conocimiento de ellas para completar el sentido del texto.

En el primer párrafo del artículo, por ejemplo, nos informa que, además de la ilustración de la carrera de carros en honor de Patroclo, Clitias ha representado en el Vaso François el mito de Troilo, ausente en la Ilíada. Por lo tanto, en la página siguiente, cuando nos dice que en ese objeto también se encuentra pintada la expedición de Teseo a Creta, que tampoco forma parte de la Ilíada (aunque él en ese caso no lo mencione), la pregunta que deberíamos hacernos es: ¿Hay algo más dibujado en esa cratera?; o bien: ¿Podemos confiar en la secuencia narrativa que ofrece este autor? ${ }^{18} \mathrm{Al}$ retroceder, porque eso es lo que Ahl nos propone, avanzar y releer, guipar y repensar, notamos que en la primera nota al pie está la información necesaria para conocer el resto de las imágenes del vaso. No obstante, lo único que aprenderemos leyendo, además de que la primera versión del artículo fue escuchada en Londres, ${ }^{19}$ es que Clitias no representa a Diomedes ganando esa carrera de carros (como ocurre en la

16. Cfr., por ejemplo, Ahl (1985, p. 201-211;1989;2002); Ahl y Roisman (1996), particularmente el apartado introductorio; y Ahl (2012, p. 281): "Sophocles' special twist is having the mythic Oedipus investigate that which, as a mythic being, he does not have: a personal past. He has only the numerous and conflicting narrations of himself in earlier narratives".

17. Cfr. Ahl (2010, p. 11): "He has also taught us how to read his own prose. Quintilian is as adept at crafting complex figured usages for his own didactic purposes as he is at noting their presence and analyzing them in the works of others". 18. Cfr. Ahl (2012, p. 285): "Our training, following the Christian tradition, inclines us to believe in authority when it comes to the literary written word, and to accept, as do Oedipus and Frederic, what their fellow characters tell them without investigating their motives".

19. El título original, que sugiere un politeísmo menos enfático, figura en el programa de actividades del encuentro académico donde fue leído y puede consultarse en el sitio web de la Sociedad Literaria de Londres: http://literarylondon. org/conference/LL2012.pdf. 
Ilíada), sino ocupando el tercer puesto, detrás de Odiseo y de Automedonte. ${ }^{20} \mathrm{Al}$ contrastar lo escrito en las obras citadas, los paralelismos propuestos por los recortes y omisiones narrativas presentes en el artículo comienzan a derrumbarse. De repente ya no está del todo claro si Maradona, como Antíloco, hizo trampa, o si solo jugó, como un profesional, llevando al límite las reglas deportivas vigentes. ${ }^{21}$ ¿Acaso no han afirmado otros lo mismo acerca de los límites fijados por la zona de exclusión total, considerando un estratégico movimiento político y militar el hundimiento del Crucero ARA General Belgrano? De repente, tampoco parece tan claro que el Reino Unido, como Diomedes, haya carecido de ayuda norteamericana durante la guerra de Malvinas: ¿ganaría de nuevo sin la mano de Atenea? ${ }^{22} \mathrm{~A}$ su vez, en términos deportivos, ¿no hubo excesivas manos alterando las contiendas futbolísticas de los campeonatos mundiales de 1966 y 1978? (cfr. Boccalatte, 05/06/20020; Mateo, 02/02/2021). Y si los dioses solo intervienen con justicia, para favorer el triunfo de quienes ya de por sí merecen ganar (cfr. Willcock, 1976, p. 256-257), ¿por qué el poder de Menelao, Stanley Rous, Joao Havelange o Ronald Reagan, parece haber alterado tantas veces los resultados?

Para intentar comprender esas disputas (disputes es la primera palabra del artículo de Ahl), hay que saber mirar (seen es la última palabra del artículo de Ahl) por uno mismo e interpretar más allá de lo que cualquier narrador diga de manera explícita. Homero opta, cuidadosamente, por no describir lo que ocurre en el desarrollo mismo de la carrera de carros, solo sabemos que Idomeneo y Áyax discuten acerca de lo que ven, sin lograr ponerse de acuerdo respecto a la primacía de Diomedes o Eumelo, hasta que Aquiles los interrumpe y dice que no tiene importancia saber a quién le asiste la razón, porque el resultado de la contienda dirimirá el asunto (cfr. Peradotto, 1990, p. 110, n. 11). Del mismo modo que Homero, Frederick Ahl prefiere que el lector observe las discrepancias y saque sus propias conclusiones, que afine la dirección de la mirada, por ejemplo, hacia las otras dos ilustraciones del Vaso François a las que solo aludió de manera oblicua: la emboscada de Aquiles a Troilo, que en esa versión tiene lugar fuera de la zona de combate; pero también la imagen de un grupo de sanguinarios y borrachos centauros, presentándoles batalla a Teseo y los lapitas.

\section{Miami, Sandwich and now Geneva}

Nada de lo afirmado y observado en los apartados anteriores, sin embargo, ocurre en la superficie textual del artículo. Para leer de ese modo hay que aceptar la ambigüedad discursiva de un trabajo que, como el autor mismo informa, no fue originalmente leído sino escuchado por sus primeros destinatarios. Debemos pensar, entonces, que tanto el encadenamiento de erratas en las notas al pie, como los juegos de palabras con los nombres del buque Sheffield y del Crucero ARA General Belgrano no fueron concebidos para la oralidad. Ante el dictado de una conferencia, no existe la posibilidad inmediata de releer los pasajes de las obras literarias comentadas, ni de buscar las imágenes de los objetos iconográficos descriptos. La concurrencia solo puede confiar en la autoridad enunciativa del orador y, en todo caso, intentar recuperar a través de sus palabras lo que vagamente recuerde de las posiciones obtenidas por los distintos competidores en los juegos fúnebres de la Ilíada.

20. No obstante, cfr. Lowenstam (1997, p. 27-28, y p. 51, n. 94).

21. Cfr. Ahl (2015b, p. 14): "because a foul, until recently, has counted only if seen live by officials".

22. Cfr. Kisiel (11/03/2010); y Winchester (14/01/2012), traducido en Winchester (16/01/2012). 
A diferencia del auditorio de Homero, para cuyos miembros la certeza de la victoria de Diomedes debía resultar evidente a partir de la enumeración misma de los contendientes (cfr. Willcock, 1973, p. 4), probablemente el público de Frederick Ahl, en junio del año 2012, sintiera más próximas las discusiones diplomáticas por la soberanía de las islas y su vinculación con el inminente desarrollo de los Juegos Olímpicos (cfr. Couzens, 09/05/2012), que la presencia o la ausencia de la intervención divina de Atenea en la carrera de carros. Para ese auditorio, la lógica indicaría que el resultado de la Guerra de Malvinas reflejó adecuadamente la correlación de fuerzas militares enfrentadas. No resultaría tan razonable, en cambio, que el segundo gol de Maradona contra Inglaterra pudiera ser considerado, en Londres, una convalidación de la justicia poética que los argentinos solemos atribuir a la conversión del primero. Si, como explica Ahl (1984a, p. 180): "People will grasp only what, in a sense, they already know", finalizar la exposición citando fragmentos de la Eneida parece haber sido una decisión adecuada. ${ }^{23}$

Cuando el texto pasa de la oralidad a la escritura, el lector dispone de muchos más recursos para demorarse en las inconsistencias, verificar las fuentes e incluso disentir críticamente con las conclusiones. No obstante, es precisamente en esa instancia cuando a la autoridad enunciativa del escritor se suma la autoridad de la superestructura del artículo académico y sus convenciones. Todo género discursivo condiciona nuestra lectura. Por esa razón, cuando en un poema aparece alguna definición científica, tiene lugar "una transmutación, un cambio de signo: la fórmula científica deja de servir a la demostración y más bien tiende a destruirla" (Paz, 1956, p. 39); inversamente, cuando la función poética del lenguaje irrumpe en un artículo académico, los recursos vinculados con la opacidad lingüística se suman a las estrategias de argumentación. ${ }^{24}$ Lo que hace etimológicamente formidable

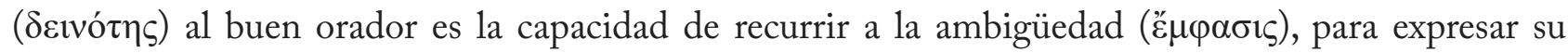
opinión libremente (aperte), mientras aparenta decir con franqueza (palam) otra cosa (cfr. Ahl, 1984a). En este caso, para lograr un efecto similar dentro de una superestructura discursiva altamente codificada, el autor parece haber considerado necesario enmascarar ciertas estrategias formales bajo un aspecto más próximo a la inspiración, que a la rigurosidad técnica del artículo científico. Ello le permite evitar asumir la función de orfebre en la trama de aparentes equívocos propuesta, delegando su responsabilidad a una sucesión de accidentes lingüísticos, imprecisiones y errores tipográficos. Con relación a este último punto, el trabajo de Frederick Ahl propone también un feraz paralelismo entre la práctica sistemática del engaño profesional en el fútbol, donde cometer ciertas infracciones y simular haber recibido otras forma parte de las habilidades deportivas inherentes al juego, ${ }^{25}$ y la conversación sobre la experticia del rapsoda, que tiene lugar entre Sócrates e Ión, en el homónimo diálogo de Platón:

As Plato's Socrates implied long ago, the assertion of divine intervention is a way of making fraud respectable. The poetic reciter Ion, demolished by Socrates' rhetoric, is asked to choose whether he wants to be reckoned a cheat or a man divinely inspired: "Choose whether you want us to reckon

23. Cfr. Ahl (2010, p. 12): "Virgil allows several simultaneous readings (...) and does not force the reader to privilege any. One can quote safely (and with approval) from many parts of the Aeneid under even the most oppressive regimes whether one is in the company of the tyrant or of the tyrant's enemies".

24. Cfr. Ahl (2002, p. 131): "It is the process, with which we are all familiar, of making our rivals our footnotes, representing them as misinformed, wrongheaded, or simply stupid".

25. Cfr. Ahl (2015b, p. 14): "Such 'professional fouling' requires practice to elude detection; it is a darker area in a player's repertoire of footballing skills". 


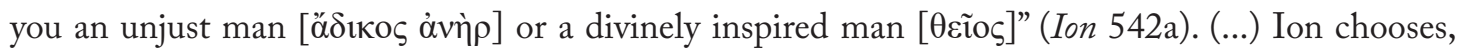

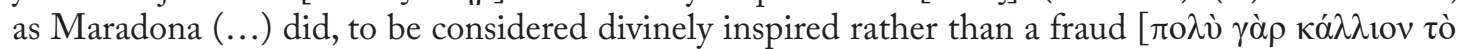
$\theta \varepsilon \tilde{\varepsilon}_{0} v$ vo $\left.\mu i \zeta \varepsilon \sigma \theta \alpha \mathrm{l}\right]$; and Socrates sarcastically, and rather smugly, concedes him the consolation of this

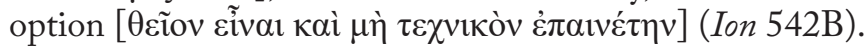

En el pasaje citado por el autor, el léxico permite homologar a quien hace trampa y no respeta

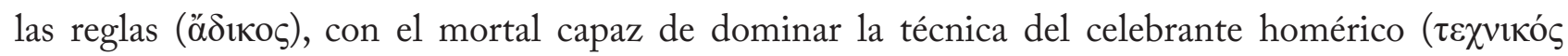

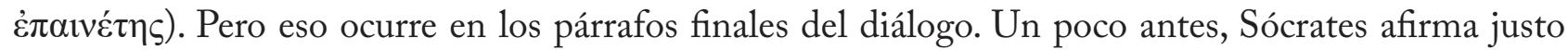

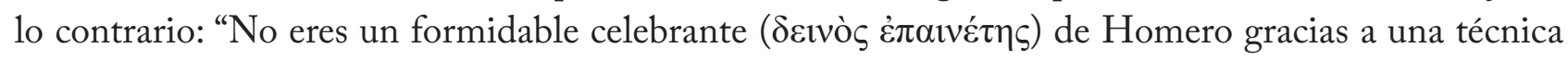

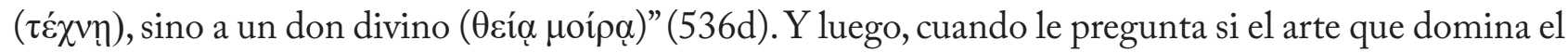

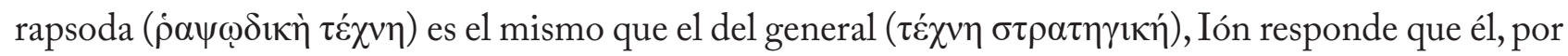

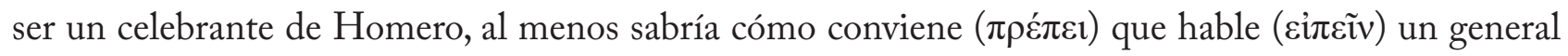

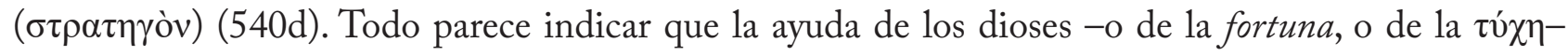
permite ennoblecer la técnica subyacente tras el despliegue de una formidable estrategia deportiva, militar o verbal. ${ }^{26}$ En el trabajo de Frederick Ahl sobre Maradona y la mano de Dios, en cambio, lo que parece configurar poéticamente el arte de la crítica es la presunción de inocencia, la falta técnica de destreza profesional en el manejo de las convenciones formales del discurso científico. Como hemos intentado demostrar, se trata de un artículo donde un número de página equivocado, la duplicación de una nota al pie, un moroso error tipográfico, el uso fortuito del lenguaje inclusivo, e incluso la letal alteración de una palabra pueden transformarse en picaportes parlantes, más propios de Alicia en el País que de nuestros claustros. Lo más prudente, en todo caso, será siempre dejar gambetear al autor y contemplar el milagro.

Sobredosis de talento, convertía a los rivales en estatuas de cemento

Las Pastillas del Abuelo

\section{Referencias bibliográficas}

Ahl, F. (1984a). The Art of Safe Criticism in Greece and Rome. The American Journal of Philology, 105 (2), 174-208.

Ahl, F. (1984b). The Rider and the Horse. Politics and Power in Roman Poetry from Horace to Statius. Aufstieg und Niedergang der römischen Welt, 32 (1), 40-124.

Ahl, F. (1985). Metaformations. Soundplay and Wordplay in Ovid and other Classical Poets. Ithaca, Cornell University Press.

Ahl, F. (1988). Ars est Caelare Artem (Art in Puns and Anagrams Engraved). En Culler, J. (ed.), On Puns. The foundation of letters (pp. 17-43). New York, Basil Blackwell.

26. Cfr. Willcock (1973, p. 4): "Pindar understood it clearly; whatever one's ability, and industry and application, final success needs something more, and the Greeks called it the hand of a god $[\theta \varepsilon \sigma \tilde{v} \pi \alpha \lambda \dot{\alpha} \mu \alpha 1]$ ". 
Ahl, F. (1989). Homer, Vergil, and Complex Narrative Structures in Latin Epic: An Essay. Illinois Classical Studies, 14 (1/2), 1-31.

Ahl, F. (2000). Quintilian and Lucan. En Homke, N.y Reitz, C. (eds.), Lucan's Bellum Civile: Between epic tradition and aesthetic innovation (pp.1-15). Berlin, Walter De Gruyter.

Ahl, F. (2002). Wordplay and apparent fiction in the Odissey. Arethusa, 35 (1), 117-132.

Ahl, F. (2007). Troy and Memorials of War. En Winkler, M. (ed.), Troy. From Homer's Iliad to Hollywood Epic (pp. 163-185). Malden, Blackwell.

Ahl, F. (2011). Translating a Paean of Praise. En Parker, J.y Mathews, T. (eds.), Tradition, Translation, Trauma. The Classic and the Modern (pp. 29-37). New York, Oxford University Press.

Ahl, F. (2012). Making Poets Serve the Established Order. Editing for Content in Sophocles, Virgil and W. S. Gilbert. Partial Answers, 10 (2), 271-301.

Ahl, F. (2015a). A mão de deus: Diego Maradona e a natureza divina da trapaça na Antiguidade Clássica. Archai: As origens do pensamiento ocidental, 14, 11-19.

Ahl, F. (2015b). Transgressing Boundaries of the Unthinkable. Sophocles, Ovid, Vergil, Seneca, and Homer Refracted in Statius' Thebaid. En Dominik, W. J.; Newlands, C. E. y Gervais, K. (eds.), Brill's companion to Statius (pp. 240-265). Leiden, Koninklijke Brill.

Ahl, F. y Roisman, H. M. (1996). The Odyssey Re-Formed. Ithaca, Cornell University Press.

Aldous, R. (2012a). Reagan and Thatcher: The Difficult Relationship. London, Random House.

Aldous, R. (2012b). Reagan and Thatcher: The Difficult Relationship. New York, W. W. Norton \& Company.

Baños Pozzati, M. (6 de diciembre de 2020). The Wolfe Tones, el legendario grupo irlandés que les canta a las Islas Malvinas Argentinas y está prohibido en Gran Bretaña. Diario Clarín. https://bit.ly/3gZtUBX.

Boccalatte, J. (5 de junio de 2020). Argentina 6-Perú 0, uno de los partidos más polémicos en la historia de los mundiales. Telam. https://bit.ly/2SSYs0p.

Corominas, J. (1983). Diccionario Crítico Etimológico Castellano e Hispánico (vol. 6: RI-X). Madrid, Gredos.

Couzens, G. (9 de mayo de 2012). Embarrassment for Argentina as it emerges athlete star of controversial Falklands ad WON'T be selected for Olympics. Dailymail. https://bit.ly/3dbV9YZ.

Deutscher, G. (2010). El prisma del lenguaje. Cómo las palabras colorean el mundo. Barcelona, Ariel.

Fantham, E. (1995). Recent Readings of Ovid's Fasti. 'Il Poeta e il Principe: Ovidio e il Discorso Augusteo' by Alessandro Barchiesi; 'Ovid and the Fasti: A Historical Study' by Geraldine HerbertBrown. Classical Philology, 90 (4), 367-378.

Gagarin, M. (1983). Antilochus'Strategy: The Chariot Race in Iliad 23. Classical Philology, 78 (1), 35-39. 
Galego-Díaz, S. (4 de mayo de 1982). Irlanda pide a la Comunidad Económica Europea que retire sus sanciones contra Argentina. El Pais. https://bit.ly/2Uqc97c.

Goñi, U. (6 de marzo de 2012). Roger Waters softens Falkland remarks. The Guardian. https://bit.ly/3vQfXvz.

Guesdon, J. M. y Margotin, P. (2017). Pink Floyd. All the songs. The story bebind every track. New York, Black Dog \& Leventhal.

Jakobson, R. (1975). La lingüística y la poética. En Ensayos de lingüística general (pp. 347-395). Barcelona, Seix Barral.

Jebb, R. (1894). Sophocles: The Plays and Fragments, with critical notes, commentary, and translation in English prose. Part VI: The Electra. Cambridge, Cambridge University Press.

Kisiel, R. (11 de marzo de 2010). Falkland Islands? Or the Malvinas, says top U.S. official in snub to Britain over oil row. Dailymail. https://bit.ly/3gS974m.

Kyle, D. (2007). Sport and Spectacle in the Ancient World. Oxford, Blackwell.

Lowenstam, S. (1997). Talking Vases: The Relationship between the Homeric Poems and Archaic Representations of Epic Myt. Transactions of the American Philological Association, 127, 21-76.

Ludmer, J. (2000). El género gauchesco. Un tratado sobre la patria. Buenos Aires, Perfil. (Original de 1988.)

Mateo, J. J. (2 de febrero de 2021). Maradona y la revancha mundial. Diario Prensa Libre. https://bit.ly/2SmlBrA.

McSmith, A. (8 de abril de 2008). Aryan ideals, not ancient Greece, were the inspiration behind flame tradition. Irish Independent. https://bit.ly/2UBsCFR.

McSmith, A. (23 de octubre de 2011). Aryan ideals, not ancient Greece, were the inspiration behind flame tradition. The Independent. https://bit.ly/3gSJOPW.

Moreno, O. (2010). La construcción de la Nación Argentina. El rol de las Fuerzas Armadas. Debates Históricos en el Marco del Bicentenario 1810-2010. Buenos Aires, Ministerio de Defensa.

Moro, R. O. (1985). La guerra inaudita. Historia del conflicto del Atlántico Sur. Buenos Aires, Fuerza Aérea Argentina.

Moro, R. O. (1989). The History of the South Atlantic Conflict: The War for the Malvinas. New York, Praeger.

Moro, R. O. (2005). La trampa de Malvinas. Parte I. Historia del conflicto del Atlántico Sur. Buenos Aires, Edivern.

O'Connell, H. (29 de diciembre de 2012). Falklands War: Irish response to Belgrano sinking drew British anger. The Journal. https://bit.ly/3xMhPqE.

O'Connell, H. (2 de abril de 2019). Guerra de las Malvinas: la respuesta de Irlanda al hundimiento del Belgrano que provocó la furia británica. The Southern Cross. https://bit.ly/3zTjsVo. 
Paz, O. (1956). El arco y la lira. México, Fondo de Cultura Económica.

Peradotto, J. (1990). Man in the middle voice. Name and Narration in the Odissey. Princeton, Princeton University Press.

Richardson, N. (1993). The Iliad. A Commentary. Volume VI: books 21-24. Cambridge, Cambridge University Press.

Shackleton Bailey, D. (2019). Marcus Annaeus Lucanus. De Bello Civili Libri X. Berlin, De Gruyter.

Steinsleger, J. (2 de diciembre de 2020). Diego Armando Maradona (1960-2020). Diario La Jornada. https://bit.ly/3q1OYqJ.

Wachter, R. (1991). The inscriptions of the Francois Vase. Museum Helveticum, 48 (2), 86-113.

Waters, R. (5 de marzo de 2012). Roger Waters negó haber afirmado "categóricamente" que las Malvinas son argentinas. La Nación. https://bit.ly/3jarSBW.

Willcock, M. (1973). The Funeral Games of Patroklos. Bulletin of the Institute of Classical Studies, 20, $1-11$.

Willcock, M. (1976). A Companion to The Iliad. Based on the Translation by Richmond Lattimore. Chicago, University of Chicago Press.

Willcock, M. (1988). Homer's Chariot Race and Vergil's Boat Race. Proceedings of the Virgil Society, 19,1-13.

Winchester, S. (14 de enero de 2012). One Falklands problem, one civilized solution. The Times. https://bit.ly/3qp9Op9.

Winchester, S. (16 de enero de 2012). El problema de Malvinas. El territorio. https://bit.ly/3vT5G1M.

Ziogas, I. (2016). Introduction: Power, Puns and Politics From Horace to Silius Italicus. En Mitsis, P. y Ziogas, I. (eds.), Wordplay an Powerplay in Latin Poetry (pp. 1-12). Berlin, De Gruyter.

*Gabriela Andrea Marrón es Profesora en Letras, Licenciada en Letras y Doctora en Letras por la Universidad Nacional del Sur. Es asistente de docencia en las materias Cultura Clásica y Lengua y Cultura Latina I (Departamento de Humanidades, UNS). Es investigadora adjunta del CONICET. Sus áreas de interés son el género épico, la literatura latina de la tardía Antigüedad y sus relaciones con las teorías sobre la intertextualidad y los estudios sobre la traducción literaria.

RECIBIDO: 29/04/2021

ACEPTADO: 18/06/2021 